\title{
On the trail of primate scent signals: A field analysis of callitrichid scent-gland secretions by portable gas chromatography-mass spectrometry 00
}

\author{
Alice C. Poirier ${ }^{1,2}$ (1) | John S. Waterhouse ${ }^{2}$ | Mrinalini Watsa $^{3,4}$ | \\ Gideon A. Erkenswick ${ }^{4,5,6}$ | Laís A. A. Moreira ${ }^{1}$ | Jia Tang ${ }^{1}$ | Jacob C. Dunn ${ }^{2,7,8}$ | \\ Amanda D. Melin ${ }^{1,9}$ | Andrew C. Smith ${ }^{2}$
}

\author{
${ }^{1}$ Department of Anthropology and Archaeology, University of Calgary, Calgary, Canada \\ ${ }^{2}$ School of Life Sciences, Anglia Ruskin University, Cambridge, UK \\ ${ }^{3}$ Population Sustainability, Institute for Conservation Research, San Diego Zoo Global, San Diego, California, USA \\ ${ }^{4}$ Field Projects International, Saint Louis, Missouri, USA \\ ${ }^{5}$ Department of Biology, University of Missouri St. Louis, Saint Louis, Missouri, USA \\ ${ }^{6}$ Department of Molecular Microbiology, Washington University School of Medicine, Saint Louis, Missouri, USA \\ ${ }^{7}$ Biological Anthropology, Department of Archaeology, University of Cambridge, Cambridge, UK \\ ${ }^{8}$ Department of Cognitive Biology, University of Vienna, Vienna, Austria \\ ${ }^{9}$ Department of Medical Genetics and Alberta Children's Hospital Research Institute, University of Calgary, Calgary, Canada
}

\section{Correspondence}

Alice C. Poirier, Department of Anthropology and Archaeology, University of Calgary, 2500 University Drive NW, T2N 1N4, Calgary, AB, Canada.

Email: alice.poirier@ucalgary.ca

\section{Funding information}

Canada Foundation for Innovation; Canada Research Chairs; Field Projects International; Anglia Ruskin University,

Grant/Award Number: PhD Scholarship; Primate Society of Great Britain,

Grant/Award Number: Research Grant; Natural Sciences and Engineering Research Council of Canada, Grant/Award Number: 2017-03782

\begin{abstract}
Chemosignals are mediators of social interactions in mammals, providing con- and hetero-specifics with information on fixed (e.g., species, sex, group, and individual identity) and variable (e.g., social, reproductive, and health status) features of the signaler. Yet, methodological difficulties of recording and quantifying odor signals, especially in field conditions, have hampered studies of natural systems. We present the first use of the Torion ${ }^{\circledR}$ portable gas chromatography-mass spectrometry (GC-MS) instrument for in situ chemical analysis of primate scents. We collected and analyzed swab samples from the scent glands and skin from 13 groups (57 individuals) of two sympatric species of wild emperor tamarins, Saguinus imperator, and Weddell's saddleback tamarins, Leontocebus weddelli (Callitrichidae). In total, 11 compounds of interest (i.e., probably derived from the animals) could be detected in the samples, with 31 of 215 samples containing at least one compound of interest. The composition of these 31 samples varied systematically with species, group, sex, and breeding status. Moreover, we tentatively identified seven of the compounds of interest as methyl hexanoate, benzaldehyde, ethyl hexanoate, acetophenone, a branched C15 alkane, 4-methoxybenzaldehyde, and hexadecan-1-ol. As the field of primate semiochemistry
\end{abstract}


continues to grow, we believe that portable GC-MS instruments have the potential to help make progress in the study of primate chemosignaling in field conditions, despite limitations that we encountered. We further provide recommendations for future use of the Torion ${ }^{\circledR}$ portable GC-MS for in situ analyses.

\section{KEYWORDS}

Callitrichidae, chemosignaling, portable GC-MS, scent-marking, semiochemicals, solid-phase microextraction

\section{1 | INTRODUCTION}

We have recently witnessed advances in the understanding and development of analytical methods for the study of semiochemicals-the chemical means of communication used by living species-in a range of vertebrate taxa (Müller-Schwarze, 2006; Wyatt, 2014). In humans, for example, there have been recent advances relevant to medicine and human evolution (e.g., chemosensory signals of major histocompatibility complex-dependent mate selection, Havlíček \& Roberts, 2009; Penn \& Potts, 1998; Winternitz \& Abbate, 2015; and of mother-infant recognition, Doucet et al., 2009; Vaglio, 2009). These findings led to the emergence of research on primate chemosignaling, at behavioral (e.g., Greene \& Drea, 2014; Henkel \& Setchell, 2018), chemical (e.g., Scordato et al., 2007; Setchell et al., 2010; Weiß, Kücklich, et al., 2018), and genetic levels (e.g., Knapp et al., 2006; Setchell et al., 2011). Yet, our knowledge of primate chemosignaling is still limited (Heymann, 2006a). This is primarily due to the methodological difficulties of recording and quantifying odor cues and signals, especially in field conditions, and the relative inconspicuousness of olfactory-related behaviors in primates, compared with auditory and visual behaviors (Drea et al., 2013; Wyatt, 2015).

The development of modern analytical chemistry techniques has improved our ability to investigate a wide range of semiochemicals (e.g., of various molecular weights, polarities, and chemical classes) from the scent-gland secretions and body odors of numerous mammalian species (see reviews by Apps et al., 2015; Burger, 2005). However, state-of-the-art semiochemistry techniques do not resolve the common challenges faced by field biologists when studying animals in the wild, which revolve around the collection, storage, and transportation of samples. At present, two approaches are used in field semiochemistry. The first approach is to collect odor samples via swabs (e.g., Leclaire et al., 2017; Weiß, Kücklich, et al., 2018) or thermal desorption tubes (e.g., Kücklich et al., 2017; Weiß, Marcillo, et al., 2018), or to collect deposited secretions (e.g., scent marks left on a surface, T. E. Smith et al., 2001), or excretions such as urine (e.g., DelBarco-Trillo et al., 2011) directly into a container, and transport them to a laboratory for analysis by chromatography. Though thermal desorption tubes can be transferred from the field to the laboratory without any need for cold shipment, swabs, secretions, and excretions have to be stored at a sufficiently low temperature, which can be challenging (Drea et al., 2013). The second approach is to extract and analyze semiochemicals in situ using portable devices such as an electronic nose, which can identify an odor by matching the response pattern of an array of gas sensors to a known odor pattern (Nagle et al., 1998; Persaud \& Dodd, 1982). Electronic noses are notably used in human clinical research, such as for screening diseases in body odor and breath (reviewed in Röck et al., 2008). However, these instruments are limited to preset odor patterns and do not allow discrimination between individual compounds, which can be challenging in field-based semiochemistry, where the range and identities of compounds contributing to odors are still largely unknown. More recently, several chromatography companies have begun developing miniaturized gas chromatography-mass spectrometry (GC-MS) devices, which can potentially conduct full chemical analyses of volatile components in remote conditions. These devices were originally developed for screening specific volatiles in the fields of environmental science (Feijó Barreira et al., 2015; Gałuszka et al., 2015), food manufacturing (Jjunju et al., 2019), and chemical warfare (Diken et al., 2012), but are now starting to be used in the field of animal chemosignaling. For example, the Inficon Hapsite ${ }^{\circledR}$ portable GC-MS device was used for the analysis of the body odor of common marmosets, Callithrix jacchus, in both captive (Kücklich et al., 2017) and wild (Thompson et al., 2020) conditions. According to the authors, this portable GC-MS device, though less sensitive than benchtop models, represents a viable alternative to laboratory-based methods for the analysis of samples under field conditions (Thompson et al., 2020).

Researchers are increasingly recognizing the prominent role of chemosignaling in primates' social and reproductive systems (Drea, 2015; Snowdon et al., 2006). Semiochemicals produced by the sender are liberated in the environment, either via passive exudation of body odors or in excretions, or via active deposition of scent-gland secretions during scent-marking, and can constitute inter- and intraspecific cues and signals (Wyatt, 2014). Chemical profiles of scentgland odor secretions can convey information on species (e.g., in owl monkeys, Aotus spp., Spence-Aizenberg et al., 2018), group and sex (e.g., in mandrills, Mandrillus sphinx, Setchell et al., 2010; Vaglio et al., 2016), breeding status (e.g., in Coquerel's sifakas, Propithecus coquereli, Greene \& Drea, 2014; and in cotton-top tamarins, Saguinus oedipus, Washabaugh \& Snowdon, 1998), and individual identity (e.g., in female common marmosets, T. E. Smith et al., 2001). Furthermore, primate specialized scent-glands may also contain different semiochemicals, allowing different cues and signals to be conveyed via one or another of the scent-glands, as suggested in ring-tailed lemurs, Lemur catta (Greene et al., 2016; Scordato et al., 2007). 


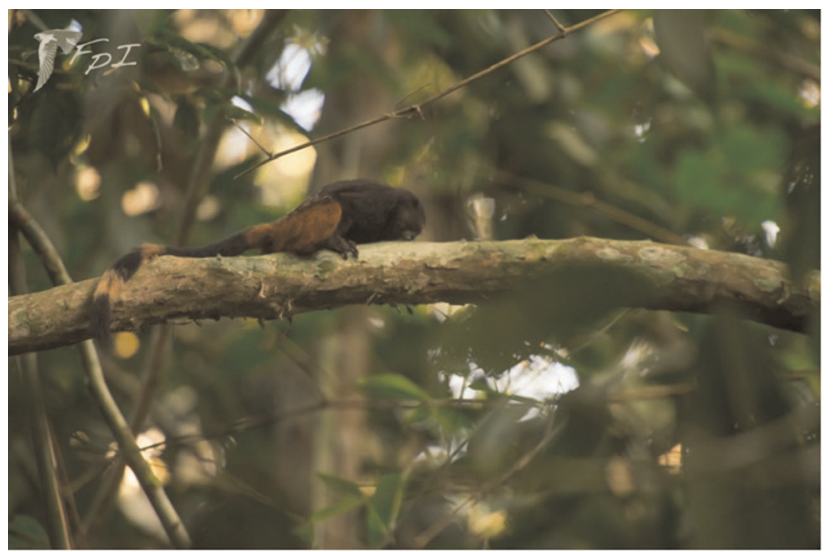

FIGURE 1 Adult male Weddell's saddleback tamarin, Leontocebus weddelli, depositing a suprapubic scent-mark on a branch. The animal's tail was bleached in parts during an annual capture-and-release to facilitate individual differentiation. Photo credit: Field Projects International

Callitrichids (Callitrichidae, Neotropical primates) are an excellent model family for the study of primate chemosignaling, as they rely heavily on odor signals and possess a well-developed vomeronasal organ. The vomeronasal organ, part of the accessory olfactory system specialized in the detection of nonvolatile chemosignals, is known to be at least partially functional in callitrichids, unlike in other primate taxa (Evans, 2006; T. D. Smith et al., 2011). The family Callitrichidae ranges from Panama to southern Brazil and is found in a variety of habitat types, from tall primary forests to farmlands (Sussman, 2003). In callitrichids, olfactory cues and signals are secreted by three specialized scent-glands on the anogenital, suprapubic, and sternal areas of the body, which they conspicuously deposit on branches and lianas in their environment (Figure 1; Epple et al., 1993). Conspecifics investigate each other's scent marks by sniffing, muzzle-rubbing, licking, or overmarking scented spots (T. E. Smith et al., 1997). Several functions have been attributed to callitrichid scent-marking behavior, including the advertisement of identity, reproductive and dominance status; territorial advertisement and defense; and spatial orientation and signaling of food resource location (Epple, 1986; Lazaro-Perea et al., 1999; Miller et al., 2003; reviewed in Heymann, 2006b).
Here, we present results from the first field use of the Torion ${ }^{\circledR}$ portable GC-MS (PerkinElmer, 2016) utilized for the analysis of callitrichid scent-gland secretion samples in situ. This study aims to: (1) Assess the success of the use of the Torion ${ }^{\circledR}$ GC-MS for the analysis of primate semiochemicals, and provide advice for future field research using this device; (2) investigate differences in the chemical composition of wild callitrichid scent-gland secretions, at the levels of species, groups, sex and breeding status, and between scent-gland type; and (3) examine the putative identity of the compounds retrieved from these wild samples. We end by commenting on the relevance of these contributions for advancing method and theory in primate chemosignaling.

\section{2 | METHODS}

\section{1 | Study subjects}

We studied two sympatric callitrichid species, the bearded emperor tamarin, Saguinus imperator subgrisescens (Hershkovitz, 1979, further referred to as $S$. imperator) and the Weddell's saddleback tamarin, Leontocebus weddelli (formerly S. fuscicollis weddelli, Deville 1849, recently reassigned; Buckner et al., 2015), in June 2017 at Estación Biológica Los Amigos (EBLA) in southeastern Peru (12 $\left.{ }^{\circ} 34^{\prime} \mathrm{S}, 70^{\circ} 05^{\prime} \mathrm{W}\right)$. We capitalized on an annual capture-and-release program of both species of tamarins at EBLA, led by Field Projects International (FPI; detailed protocol in Watsa et al., 2015), to obtain swabs of tamarin scent-glands while the animals were anesthetized. A total of 24 emperor tamarins (five groups) and 33 saddleback tamarins (eight groups) were captured and sampled (Tables 1 and S1). FPI's research is conducted with annual authorization from the Peruvian Ministry of the Environment (SERFOR), as well as the Animal Care and Use Committees of the University of Missouri, St. Louis (IACUC protocol \# 1208181-4), and the University of Calgary (ACC protocol \# AC15-0161). This project was approved by the Faculty of Science and Engineering Departmental Research Ethics Panel at Anglia Ruskin University. It adheres to the American Society of Primatologists Principles for the Ethical Treatment of Non-Human Primates and follows the Animal Behavior Society Guidelines (2018) and Sikes and the American Society of Mammalogists' Guidelines on Wild Mammals in Research (2016).

TABLE 1 Group composition and samples used in the present analysis

\begin{tabular}{|c|c|c|c|c|c|}
\hline Species & No. of groups & Sex & No. of individuals & No. of samples & \\
\hline \multirow[t]{2}{*}{ Saddleback tamarin, Leontocebus weddelli } & 8 & o & 22 & & 85 \\
\hline & & ᄋ & 11 & & 42 \\
\hline \multirow[t]{2}{*}{ Emperor tamarin, Saguinus imperator } & 5 & 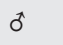 & 10 & & 39 \\
\hline & & 우 & 14 & & 49 \\
\hline \multirow[t]{3}{*}{ Total individuals in analysis } & & & 57 & Total animal samples & 215 \\
\hline & & & & Air and blank samples & 63 \\
\hline & & & & Total samples & 278 \\
\hline
\end{tabular}




\subsection{Determination of breeding status}

We determined the breeding status (i.e., primary breeders, secondary breeders, and nonbreeders) of all the study subjects using a multivariate predictive model developed by Watsa et al. (2017). Primary breeders were reproductively active males and females, regardless of whether they sired offspring. Secondary breeders were both males and females aged 1- to 2-years old and sexually mature, but not having bred yet. Nonbreeders were all offspring below 1 year of age and sexually immature (Watsa, 2013). The model used indices of reproductive morphology (i.e., vulvar index, testicular volume, suprapubic gland area, and mean nipple length), combined with known breeding status of a subset of animals in the population obtained by direct observation of copulation, to predict the breeding status of all other individuals.

\section{3 | Scent sample collection, extraction, and chemical analyses}

We collected 278 samples from the scent-glands and bodies of 57 tamarins (13 groups in total), as well as the ambient air, and blanks, over 20 days in June 2017 (Tables 1 and S1). The sampling procedure consisted of gently wiping a sterile cotton bud (Fisherbrand ${ }^{\mathrm{TM}}$ ) over the scent-gland area 10 times in an up-and-down motion while wearing nitrile gloves (online protocol: dx.doi.org/10.17504/protocols.io.brpqm $5 \mathrm{mw})$. Cotton buds were wetted with distilled water before swabbing the animals to reduce risks of skin irritations from friction. For each animal captured, we collected swab samples from the following locations: (a) Anogenital scent gland, (b) suprapubic scent gland, (c) sternal scent gland (or sternal skin area when not visible), and (d) the animal's inner thigh skin. The inner thigh is a relatively hairless region, similar to the three scent-gland areas, but lacking glandular tissue. As it was an area relatively untouched by the researchers while taking samples and measurements of the animals, the risk of contamination at the time of swabbing was reduced. Therefore, it seemed like a good control region to use in our study. We hypothesized that glandular compounds would not be found in these control regions. In addition, we took (e) two air controls (i.e., swabs left out for $30 \mathrm{~s}$, to control for background volatiles), and (f) two blank controls (i.e., empty vial and unused swab) per capture session. An entire tamarin group was captured at each successful trapping session, giving us the opportunity to collect samples from all individuals in the group during the first phase of the sedation procedure. This allowed swab collection within 15-80 min after capture, before the animals' scent-glands risked contact with other surfaces, which could have contaminated the swabs. Swabs were stored in brand new 4-ml glass screw-top vials fitted with a polytetrafluoroethylene/ rubber septum (Supelco), and kept for a maximum of $7 \mathrm{~h}$ in a cool Thermos ${ }^{\circledR}$ flask filled with gel packs pre-hilled in a freezer at around $0^{\circ} \mathrm{C}$ (online protocol: dx.doi.org/10.17504/protocols.io.brpqm5mw). Upon return from each capture session, samples were stored in a refrigerator at the field station at a mean temperature of $0.5 \pm 2.4^{\circ} \mathrm{C}$, recorded hourly by an automatized temperature data logger.
We analyzed the scent samples at the field station an average of $3.8 \pm 2.8$ days after collection using headspace extraction and a portable GC-MS (Torion ${ }^{\circledR}$ T-9; PerkinElmer, 2016; Figure S1) following a standardized protocol (dx.doi.org/10.17504/protocols.io.brpqm5mw). We extracted the samples individually using a $65-\mu \mathrm{m}$ solid-phase microextraction (SPME) fiber coated with divinylbenzene/polydimethylsiloxane (Custodion ${ }^{\circledR}$; PerkinElmer, 2016; Figure S1) for $2 \mathrm{~min}$ at room temperature $\left(23.9 \pm 3.1^{\circ} \mathrm{C}\right)$, after heating them for $2 \mathrm{~min}$ in a bath of simmering water (ca. $100^{\circ} \mathrm{C}$ ). We then desorbed the volatile-coated fiber at $300^{\circ} \mathrm{C}$ for $5 \mathrm{~s}$ into the injection port of the Torion ${ }^{\circledR} \mathrm{GC}$, which was equipped with a low polarity MXT-5 Low Thermal Mass capillary column. Helium was used as the carrier gas and the injection was in splitless mode, with split mode applied at $2 \mathrm{~s}$ with a ratio of $10: 1$, then $50: 1$ at $10 \mathrm{~s}$. The temperature of the column started at $50^{\circ} \mathrm{C}$, was held for $10 \mathrm{~s}$, and followed by an increase of $2^{\circ} \mathrm{C} / \mathrm{s}$ to the final temperature of $300^{\circ} \mathrm{C}$, which was held for $2 \mathrm{~s}$. The total runtime was $137 \mathrm{~s}$ per sample. Mass separation was performed by the Torion ${ }^{\circledR}$ toroidal ion trap MS under electron ionization mode at $70 \mathrm{eV}$, in full-scan mode in 45-500 mass-tocharge ratio $(\mathrm{m} / \mathrm{z})$ range. Each day, we ran the animal samples, blank and air controls, and fiber blanks (i.e., clean fiber run in the same conditions to control for carryover), in a randomized order. Although the Torion ${ }^{\circledR}$ possesses built-in data interpretation software, we interpreted the resulting chromatograms on a computer at a later date, because the Torion $®$ was being used for another project after sample analysis.

\subsection{Interpretation of analytical results}

We used the software ChemStation ${ }^{\mathrm{TM}}$ (Agilent) to perform automatic peak detection and integration, and the National Institute of Standards and Technology (NIST) mass spectral library (Shen et al., 2014) to tentatively identify the detected peaks within each GC-MS chromatogram (minimum matching factor higher than $80 \%$ ). We recorded the following for all detected peaks: Retention time, peak area, mass spectrum, and NIST identification. We only considered peaks eluted before $1.6 \mathrm{~min}$ as peaks after this time generally displayed excessively noisy baselines (i.e., greater than one-sixth of the highest peak). In addition, we removed from the analysis all peaks with an area less than $1 \%$ of the highest peak across all the chromatograms, and those that were too flat to be distinguished either from the baseline or from a neighboring peak, to reduce the risk of including noise in our results (Drea et al., 2013). We were able to identify those GC peaks of compounds that arose solely from the scent samples by a careful comparison of the chromatograms of scent samples, air and blank control samples, and fiber blanks (i.e., when the clean SPME fiber was run like a sample), and by close observation of the mass spectra obtained from the GC peaks. Compounds of interest were present in at least one of the tamarin samples and absent from all air and blank control samples. Peaks with similar retention times in different chromatograms could represent the same, or different, compounds. We decided which of the two possibilities applied by comparing the mass spectra of individual chromatographic peaks, as illustrated in Figure S2. 


\subsection{Statistical analyses}

All statistical analyses were performed in R v.4.0.0 operated in RStudio (R Core Team, 2020). We used a binomial logistic regression to test whether the delay between sample collection and analysis influenced the likelihood of detecting compounds in the samples, using the glm function in stats $\mathrm{R}$ package. We assessed differences in chemical composition between categories of samples at the levels of species, group, sex, breeding status, and sample type using a nonmetric multidimensional scaling (NMDS) ordination and analysis of similarity (ANOSIM) approach, based on the presence/absence of compounds of interest. For this we retained only samples presenting at least one compound of interest $(N=31)$. We computed a distance matrix of samples and their respective compound composition with the Bray-Curtis dissimilarity index, using the vegdist function in vegan $\mathrm{R}$ package (Oksanen et al., 2019). Then, we calculated 2-dimensional NMDS coordinates from the values of Bray-Curtis indices in the distance matrix, using the metaMDS function in vegan. These coordinates allowed for the visualization of dissimilarity between categories of samples. The stress factor associated with these coordinates, measuring the goodness-of-fit between predicted and observed values (similar to the $R^{2}$ value in a regression), was close to zero, reflecting the limited number of samples containing compounds of interest. Finally, we performed ANOSIM tests to assess whether sample chemical composition differed significantly between categories of samples. We used the ANOSIM function in vegan on the Bray-Curtis dissimilarity matrix of samples, with 999 permutations and species as strata (i.e., group, sex, breeding status, and sample type were nested into species). The number of compounds of interest detected was too limited to investigate statistically the influence of each individual compound in distinguishing between categories of samples. Because we analyzed all samples collected from the same tamarin group together, we were not able to control for effects pertaining to the day on which the analysis occurred.

\section{3 | RESULTS}

\subsection{Use of the Torion ${ }^{\circledR}$ GC-MS for the analysis of primate semiochemicals}

Visual inspection of the chromatograms of the 215 animal samples collected (from the anogenital, suprapubic, and sternal scent-glands, and the inner thigh skin) permitted the detection of a number of peaks that were absent in all the controls (e.g., the air samples, the blank samples, or the fiber blanks; Table S1 and Figure S2). The majority of these peaks, however, were poorly resolved, that is, they represented a superposition of two or more compounds of similar $\mathrm{m} / \mathrm{z}$, resulting in complex mass spectra. It was thus only possible to isolate 11 peaks representing single compounds. These compounds of interest (i.e., compounds A-K), were found in 31 of the samples, from 22 individuals (Tables 2 and S1). We did not observe a significant influence of the delay between sample collection and analysis on the likelihood of detecting one or several compounds of interest in the samples $\left(Z=-0.16, R^{2}<.01, p=.87\right)$.

\section{2 | Compounds detected in tamarin scent-gland secretions}

Compounds $\mathrm{C}, \mathrm{E}, \mathrm{F}$, and $\mathrm{K}$ appeared to be unique to a sample, whereas the rest were shared (Table 2). Only compound D was found in both tamarin species, the rest were detected in one or the other species (Table 2). Sample chemical composition differed significantly between emperor and saddleback tamarins (ANOSIM: $R=.22$, $P=.001$; Figure 2a). All groups of both species of tamarin presented at least one sample containing a compound of interest. The majority of compounds (81.8\%) were only retrieved from one or two groups; however compound $A$ was found in six out of the eight saddleback tamarin groups, and compound $\mathrm{G}$ from three out of the five emperor tamarin groups (Table 2). Within a species, the chemical composition of samples from the same group was significantly more similar than that of samples from different groups $(R=.46, p=.001)$. Compounds $\mathrm{C}, \mathrm{E}$, and $\mathrm{F}$ were only detected in males; compounds I, J, and $\mathrm{K}$ in females (Table 2). Within a species, sample chemical composition differed significantly between males and females $(R=.12, P=.008$; Figure 2b). Most of the compounds (63.6\%) were exclusively found in primary breeders $(N=23$ samples); three compounds were also present in samples from secondary breeders; and compound $\mathrm{F}$ was only found in a secondary breeder (Table 2). Compound A was unique in being found principally in secondary breeding males. No compound of interest was present in nonbreeders. Within a species, sample chemical composition differed marginally between primary and secondary breeders ( $R=.07, P=.053$; Figure $2 \mathrm{c}$ ). Finally, four compounds were only detected on anogenital gland swabs, two compounds only on suprapubic gland swabs, and one compound on a sternal gland swab. Compounds $\mathrm{A}, \mathrm{B}$, and $\mathrm{H}$ were retrieved from all areas sampled, including the skin, suggesting a nonspecialized glandular compound, or even an exogenous origin (Table 2). Sample chemical dissimilarity between anogenital, suprapubic and sternal gland swabs, and skin was nonsignificant $(R=.07, p=.146$; Figure $2 d)$.

\subsection{Identity of putative tamarin semiochemicals}

Automatic search in the NIST mass spectral library, together with a visual inspection of the mass spectra and retention times of the peaks of interest, allowed us to make a tentative identification of seven of the compounds characterized by these peaks: Methyl hexanoate $[B]$, benzaldehyde $[D]$, ethyl hexanoate $[E]$, acetophenone $[F]$, a branched C15 alkane [G], 4-methoxybenzaldehyde [I], and hexadecan-1-ol $[\mathrm{K}]$. We were not able to suggest identities for compounds $\mathrm{A}, \mathrm{C}, \mathrm{H}$, and J, due to high peak impurity (i.e., the peaks detected by the GC-MS were not fully resolved, hence their mass spectra were likely composed of more than one compound that were impossible to tell apart). 


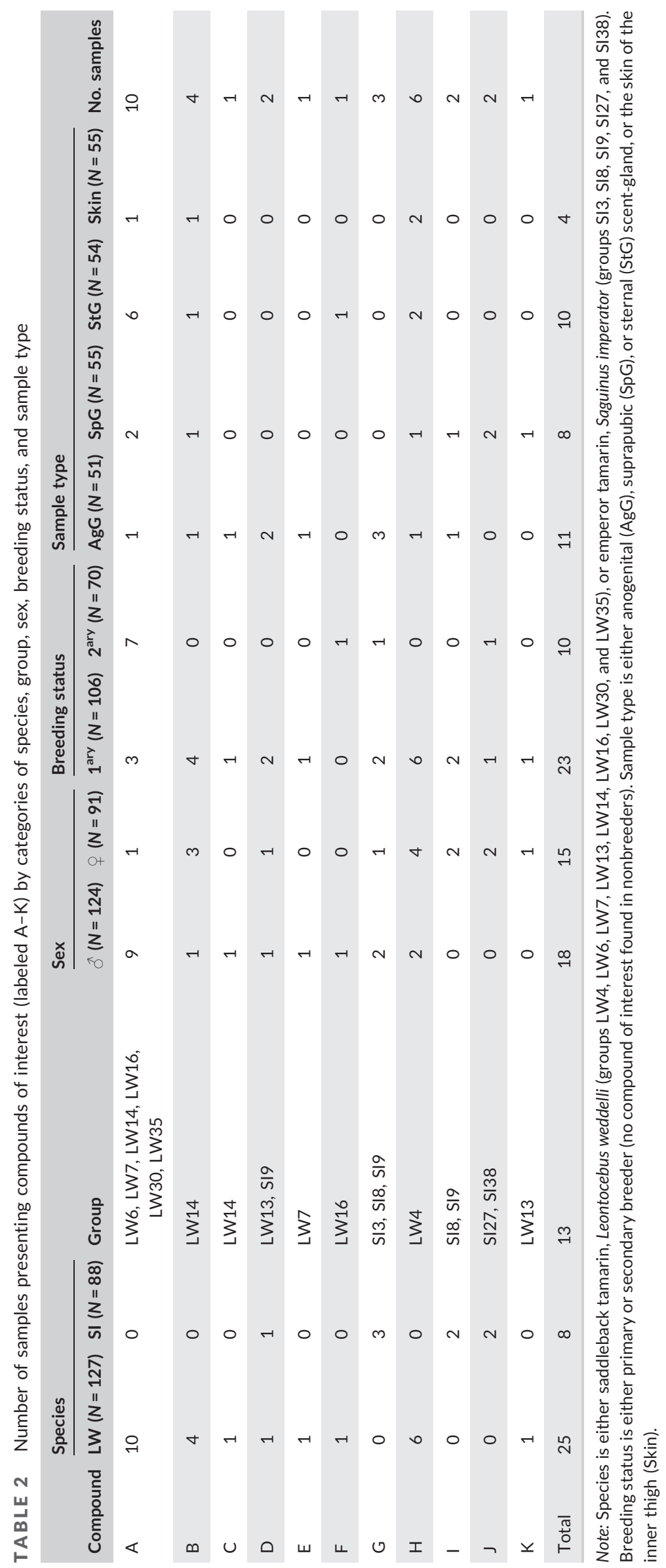


(a)

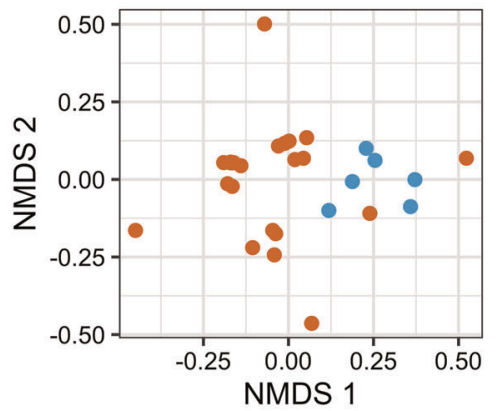

(c)

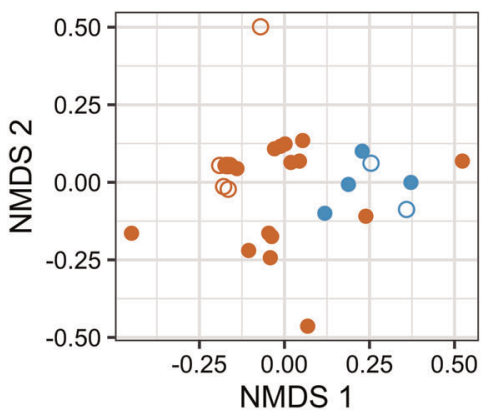

(b)

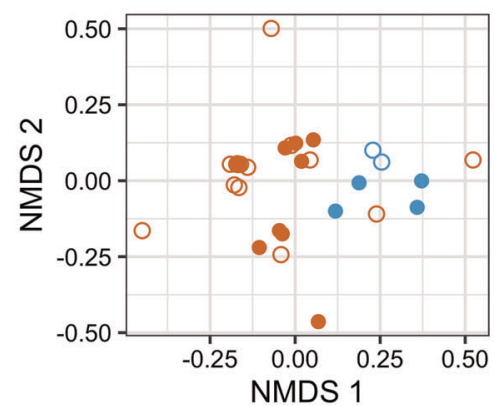

Sex

- Female

- Male
Breeding

status

- Primary

O Secondary

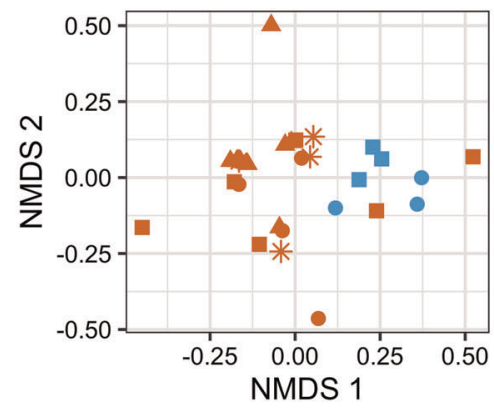

Sample

type

- Gland_AG

- Gland_SP

- Gland_ST

* Skin

FIGURE 2 Nonmetric multidimensional scaling (NMDS) plots showing similarity in sample chemical composition between (a) saddleback and emperor tamarins; (b) females and males of each species; (c) primary and secondary breeders of each species; and between (d) anogenital, suprapubic and sternal scent-gland, and skin samples (stress $<0.01)$. Only samples presenting at least one compound of interest $(N=31)$ are included

\section{4 | DISCUSSION}

\section{1 | Reflection on the use of the Torion® GC-MS for the analysis of primate semiochemicals}

We were able to detect 11 compounds of interest from wild tamarin scent-gland samples analyzed using the Torion® GC-MS. The use of this device for the analysis of callitrichid semiochemicals was principally motivated by its portability; it was the first attempt to analyze animal scent chemical composition using this instrument in situ. Similarly, Thompson et al. (2020), in a field analysis of the chemical composition of 64 common marmoset scent marks using the Hapsite ${ }^{\circledR}$ portable GC-MS, retrieved 40 volatile compounds, of which 12 were not present in any blanks and control samples (same conditions as our study). The number of compounds of interest retrieved from our samples ( $N=11$ compounds) was limited when compared with similar samples collected the following year and transported to a laboratory for analysis using a PerkinElmer Clarus 500 benchtop GCMS ( $N=25$ compounds; Poirier, 2019), despite the fact that over eight times as many samples were analyzed under in situ conditions ( $N=215$ samples) than under laboratory conditions ( $N=27$ samples). Kücklich et al. (2017), who compared the performance of the Hapsite ${ }^{\circledR}$ portable GC-MS to that of cotton swabs analyzed on an Agilent 5973 bench-top GC-MS, for the analysis of body odor of captive common marmosets, also found that the portable GC-MS retrieved fewer compounds. This was expected, as the extremely short GC runtime in a portable instrument (i.e., just over $2 \mathrm{~min}$ in our study) does not allow complete separation of individual compounds over time. Moreover, in our study, the majority of samples did not show any compounds of unambiguous glandular origin. We believe that several such compounds might have been masked by contaminant peaks, because of the low separation of compounds. Kücklich et al. (2017) and Thompson et al. (2020) reported the same limitation in their assessment of the Hapsite ${ }^{\circledR}$ portable GC-MS. Yet, the extremely short sample runtime enabled by these miniaturized portable GC-MS devices allows to process a large number of samples in a relatively short timeframe compared with bench-top instruments. In addition, the sampling method (i.e., swab in vial) and extraction method (i.e., SPME manual holder) might have favored the incorporation of contaminants in the chemical profiles. Nonetheless, a number of methodological aspects can readily be optimized to ensure greater success in future analyses of mammalian semiochemicals using the Torion ${ }^{\circledR}$ GC-MS. These include improving sampling procedures, to limit the incorporation of volatile contaminants during sample collection and extraction. Moreover, optimization of the instrumental working conditions (e.g., power generation, helium connection, availability of disposable material replacements), and preparation for on-site troubleshooting, will be necessary. We recommend that future users of the Torion ${ }^{\circledR}$ GC-MS in isolated field conditions ensure that they carefully plan for all the technical challenges that are likely to arise. For example, our study suffered from the fact that airline companies prohibited the 
transport of the Torion ${ }^{\circledR}$-specific battery and helium canisters, forcing us instead to rely on a fuel generator, the fumes of which were a potential contaminant, and a generic helium tank, which was costly and difficult to fit the Torion ${ }^{\circledR}$ system. We provide further examples and advice from our own experience in Table S3. Despite the obvious problems, we believe that portable GC-MS instruments have the potential to aid the study of primate olfactory communication in field conditions. In particular, these instruments could be a great asset for an in situ search for volatile compounds which have been identified in prior studies using a bench-top GC-MS, and for which the detectability using this instrument has already been verified in pilot studies (Kücklich et al., 2017). However, their overall performance for the detection of a multitude of unknown volatile and nonvolatile compounds present in small quantities on swabs appears insufficient.

\subsection{Compounds detected in tamarin scent-gland secretions}

The shortcomings of the analytical techniques discussed above limited our ability to identify compounds of interest in our samples. Despite the limitations, however, our results suggest differences in the chemical composition of the scent-gland odor secretions and skin of emperor and saddleback tamarins at the levels of species, group, sex, breeding status, and scent-gland type.

Nearly all compounds retrieved from the wild tamarin scentgland samples were only detected in one or the other species; only one (i.e., benzaldehyde [D]) was found in both species. Other studies have similarly found differences in sample chemical composition between species (e.g., in glandular secretions of owl monkeys, Spence-Aizenberg et al., 2018; urine of various strepsirrhine species, DelBarco-Trillo et al., 2011). 4-Methoxybenzaldehyde [I], which was only detected in swabs from emperor tamarins in our study, was also identified in deposited scent marks from captive emperor tamarins (Poirier, 2019), and cotton-top tamarins (Belcher et al., 1988; Poirier, 2019), suggesting that this compound might be widespread in the genus Saguinus.

Our study groups varied in composition of their scent-gland secretions. Other studies have reported group chemical signatures (e.g., in mandrills, Vaglio et al., 2016; Eurasian otters, Lutra lutra, Kean et al., 2017; Bechstein's bats, Myotis bechsteinii, Safi \& Kerth, 2003). Nevertheless, as we collected and analyzed all samples from one group at a time, it was not possible to know whether our results reflected actual group differences, or potential temporal differences arising from different times of sampling and analysis (i.e., batch effect).

Three of the compounds of interest, that is, 4-methoxybenzaldehyde [I], hexadecan-1-ol [K], and unidentified compound J, were only found in female samples; whereas three compounds, namely, ethyl hexanoate $[E]$, acetophenone [F], and an unidentified compound $\mathrm{C}$ were only detected in male samples. Hence, these putative tamarin compounds could be sex-specific. Moreover, no compound of interest was retrieved from nonbreeding individuals. Methyl hexanoate $[B]$, unidentified compound $C$, benzaldehyde [D], ethyl hexanoate $[E]$, unidentified compound $H$, 4-methoxybenzaldehyde [I], and hexadecan-1-ol $[\mathrm{K}]$, which were exclusively retrieved from primary breeders, may be associated with reproduction, mate choice and/or intrasexual competition in this cooperative breeding taxon (Heymann, 2003). Similarly, sex and breeding status differences have been reported in scent samples of other primates (e.g., in genital secretions of Coquerel's sifakas, Greene \& Drea, 2014; subcaudal secretions of owl monkeys, A. nancymaae, MacDonald et al., 2008; and sternal gland secretions of mandrills, Setchell et al., 2010). However, the present results may be an artifact of the small number of positive samples (i.e., samples from which at least one compound of interest was identified) in the present field study. A greater sample size will be needed to be able to confirm the specificity and function of given semiochemicals.

Unidentified compound C, benzaldehyde [D], ethyl hexanoate [E], and the branched C15 alkane [G] were only found on anogenital gland swabs; unidentified compound $\mathrm{J}$ and hexadecane-1-ol $[\mathrm{K}]$ only on suprapubic gland swabs; and acetophenone [F] on a sternal gland swab. Histological analyses of the anogenital and suprapubic scentgland skin of cotton-top tamarins (Fontani et al., 2014) and the suprapubic and sternal scent-gland skin of lion tamarins, Leonthopithecus sp. (Moraes et al., 2006), showed no difference between the tissues of these different glands. The same can, therefore, be expected for the two tamarin species studied here. Similar to the present results, Scordato et al. (2007) found chemical differences between swabs of labial, scrotal, and brachial scent-glands in ringtailed lemurs; and Spence-Aizenberg et al. (2018) between swabs of pectoral and subcaudal glands in owl monkeys. Moreover, unidentified compound $\mathrm{A}$, methyl hexanoate $[\mathrm{B}]$, and unidentified compound $\mathrm{H}$ were retrieved from swabs of the inner thigh skin region of saddleback tamarins, as well as from scent-gland samples from different individuals. Tamarins spend a lot of time in physical contact with one another while sleeping in tree knots and grooming (A. C. Smith et al., 2007). They also perform allomarking, where they deposit a scent-mark on the body of a conspecific (Lledo-Ferrer et al., 2010). It is, therefore, conceivable to find a glandular compound on another body part of the animal. As all compounds found on an animal's thigh skin were also retrieved from a gland in other individuals of the same species, we decided not to rule out these chemicals from our list of compounds of interest. However, these results demonstrate that the inner thigh does not constitute a good control region to use for tamarin semiochemical studies.

\subsection{Identity of putative tamarin semiochemicals}

Of the 11 compounds of interest detected by the Torion ${ }^{\circledR}$ GC-MS, we were able to name with reasonable confidence only seven of them, containing a variety of functional groups (i.e., two aldehydes, one alkane, one alcohol, one ester, and one carboxylic acid). Despite 
our caveats on data interpretation, we believe that we have identified several compounds that may be important in tamarin semiochemical communication. Moreover, all these compounds have been reported before as candidate mammalian semiochemicals (Table S2). The wide range of primate and other mammal species, and glands or body areas producing these semiochemicals indicates a certain ubiquity of these compounds. Benzaldehyde [D], acetophenone [F], and hexadecane-1-ol [K] were particularly ubiquitous, found in over 18 different mammalian species (Table S2). Moreover, these three compounds and 4-methoxybenzaldehyde [I] were also identified in scent-mark samples from captive emperor tamarins (Poirier, 2019).

All compounds identified here are also known to be produced by plants and bacteria (https://pubchem.ncbi.nlm.nih.gov; www. pherobase.com), which suggests they might not have been directly produced by the animals. However, it is possible that a compound of plant origin, derived from diet, serves as a mammal semiochemical (Havlíček et al., 2019; Henneken et al., 2017). Moreover, commensal bacteria present in the scent glands or on the skin may take an active part in the composition of the secretions (Carthey et al., 2018; Ezenwa \& Williams, 2014). In addition, some compounds of plant origin may have been incorporated into the swabs during sampling and correspond to the odor of the substrate branches themselves, or to contamination of the surface swabbed by food remains and/or excrement. This gives a further reason to be cautious about the chemical analyses presented here, the identity of compounds of interest, as well as their potential specificity across categories of samples (e.g., species- or sex-specific).

Some of the confounding aspects of our chemical analyses could be reduced by replicated analyses of samples. However, even two swabs taken consecutively from the same gland area might contain different compounds, as the scent-gland is continuously producing secretions. Ideally, the identity of a certain compound in a natural secretion should be validated by replicating the chemical analysis using the authentic compound. Future work with the Torion ${ }^{\circ}$ GC-MS will include a validation step in the laboratory using authentic compounds.

Additional evidence for the role of a compound as a semiochemical could be sought by performing a behavioral bioassay to assess the response of conspecifics to this particular compound. A given compound may be genuinely a component of glandular secretion, and yet show no signaling role, that is, not be a semiochemical. Such bioassays have been carried out in captive primates, such as in common marmosets (Kücklich et al., 2019; T. E. Smith \& Abbott, 1998), ring-tailed lemurs (Scordato \& Drea, 2007), and Japanese macaques, Macaca fuscata (Rigaill et al., 2017). In natural conditions, however, conducting behavioral bioassays is more challenging. Thanks to the habituation work carried out by FPI at EBLA, future work on chemosignaling in this callitrichid population will include behavioral bioassays.

\section{ACKNOWLEDGMENTS}

We acknowledge the support of the Amazon Conservation Association (USA), the Asociación para la Conservación de la Cuenca Amazónica (Peru), and the Dirección General Forestal y de Fauna
Silvestre (Peru). We would like to thank Bill Hahn (PerkinElmer, USA) and Ewen Honeymann (PerkinElmer, UK) for providing us with training and assistance with the Torion ${ }^{\circledR}$. We are grateful to Stefano Vaglio for lending us equipment for data analysis, Omer Nevo for his advice on chemical interpretation, and Fabrizio Manco for his recommendations on statistical analyses. Finally, none of this field project would have been successful without the help of all the field managers, research assistants, and other people involved with Field Projects International, in particular Alex Sacco, Frances Humes, Sam López Clinton, and Ishaan Raghunandan. This study was funded by The Natural Sciences and Engineering Research Council of Canada (Amanda D. Melin [ADM] 2017-03782), The Canada Foundation for Innovation and Canada Research Chairs program (ADM), Field Projects International, Anglia Ruskin University (PhD scholarship for Alice C. Poirier [ACP]), and a Research Grant from the Primate Society of Great Britain (ACP).

\section{CONFLICT OF INTERESTS}

The authors declare that there are no conflict of interests.

\section{AUTHOR CONTRIBUTIONS}

Alice C. Poirier: Conceptualization (equal); data curation (lead); formal analysis (lead); investigation (equal); methodology (lead); writing original draft (lead). John S. Waterhouse: Supervision (equal); writing review and editing (equal). Mrinalini Watsa: conceptualization (equal); funding acquisition (equal); investigation (equal); methodology (equal); project administration (equal); writing review and editing (equal). Gideon A. Erkenswick: Conceptualization (equal); funding acquisition (equal); investigation (equal); methodology (equal); project administration (equal); writing review and editing (equal). Jia Tang: Methodology (supporting); writing review and editing (supporting). Jacob Dunn: Data curation (supporting); supervision (equal); writing review and editing (equal). Andrew Smith: Funding acquisition (equal); project administration (equal); supervision (equal); writing review and editing (equal).

\section{PEER REVIEW}

The peer review history for this article is available at https://publons. com/publon/10.1002/ajp.23236.

\section{OPEN RESEARCH BADGES}

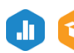

This article has earned an Open Data and Open Materials badges for making publicly available the digitally-shareable data necessary to reproduce the reported results. The data is available at https://www. ncbi.nlm.nih.gov/.

\section{DATA AVAILABILITY STATEMENT}

The data and $\mathrm{R}$ code supporting this article can be obtained from https://github.com/AlicePoirier/Poirier-et-al_AJP_2021. The protocol for this study can be found on the protocols.io online repository: https://doi.org/10.17504/protocols.io.brpqm5mw. 


\section{ORCID}

Alice C. Poirier (D) https://orcid.org/0000-0001-7947-3721

\section{REFERENCES}

Apps, P. J., Weldon, P. J., \& Kramer, M. (2015). Chemical signals in terrestrial vertebrates: Search for design features. Natural Product Reports, 32, 1131-1153. https://doi.org/10.1039/C5NP00029G

Barreira, L. M. F., Parshintsev, J., Kärkkäinen, N., Hartonen, K., Jussila, M., Kajos, M., Kulmala, M., \& Riekkola, M. L. (2015). Field measurements of biogenic volatile organic compounds in the atmosphere using solid-phase microextraction and portable gas chromatography-mass spectrometry. Atmospheric Environment, 115, 214-222. https://doi. org/10.5194/amt-11-881-2018

Belcher, A. M., Epple, G., Kuderling, I., \& Smith, A. B. (1988). Volatile components of scent material from cotton-top tamarin (Saguinus o. oedipus): A chemical and behavioral study. Journal of Chemical Ecology, 14(5), 1367-1384.

Buckner, J. C., Alfaro, J. L., Rylands, A. B., \& Alfaro, M. E. (2015). Biogeography of the marmosets and tamarins (Callitrichidae). Molecular Phylogenetics and Evolution, 82, 413-425. https://doi.org/ 10.1016/j.ympev.2014.04.031

Burger, B. V. (2005). Mammalian semiochemicals. Topics in Current Chemistry, 240, 231-278. https://doi.org/10.1007/b98318

Carthey, A. J. R., Gillings, M. R., \& Blumstein, D. T. (2018). The extended genotype: Microbially mediated olfactory communication. Trends in Ecology and Evolution, 33(11), 885-894. https://doi.org/10.1016/j. tree.2018.08.010

DelBarco-Trillo, J., Burkert, B. A., Goodwin, T. E., \& Drea, C. M. (2011). Night and day: The comparative study of strepsirrhine primates reveals socioecological and phylogenetic patterns in olfactory signals. Journal of Evolutionary Biology, 24(1), 82-98. https://doi. org/10.1111/j.1420-9101.2010.02145.x

Diken, E. G., Arno, J., Skvork, E., Manning, D., Andersson, G., Judge, K., \& Lee, E. D. (2012). Advances in field-portable ion trap GC/MS instrumentation. Chemical, Biological, Radiological, Nuclear, and Explosives (CBRNE) Sensing XIII, Proceedings of the Society for Optics and Photonics, 8358, 1-17. https://doi.org/10.1117/12.921054

Doucet, S., Soussignan, R., Sagot, P., \& Schaal, B. (2009). The secretion of areolar (Montgomery's) glands from lactating women elicits selective, unconditional responses in neonates. PLOS One, 4(10), 1-11. https://doi.org/10.1371/journal.pone.0007579

Drea, C. M. (2015). D'scent of man: A comparative survey of primate chemosignaling in relation to sex. Hormones and Behavior, 68, 117-133. https://doi.org/10.1016/j.yhbeh.2014.08.001

Drea, C. M., Boulet, M., Delbarco-Trillo, J., Greene, L. K., Sacha, C. R., Goodwin, T. E., \& Dubay, G. R. (2013). The "secret" in secretions: Methodological considerations in deciphering primate olfactory communication. American Journal of Primatology, 75(7), 621-642. https://doi.org/10.1002/ajp.22143

Epple, G. (1986). Communication by chemical signals. In G. Mitchell \& J. Erwin (Eds.), Comparative primate biology 2A: behavior, conservation, and ecology (pp. 531-580). New York: Alan R. Liss.

Epple, G., Belcher, A. M., Kuderling, I., Seller, U., Scolnick, L., Greenfield, K. L., \& Smith, A. B. (1993). Making sense out of scents: Species differences in scent glands, scent-marking behaviour, and scent-mark composition in the Callitrichidae. In A. B. Rylands (Ed.), Marmosets and tamarins: Systematics, behaviour and ecology (pp. 123-151). Oxford Science.

Evans, C. S. (2006). Accessory chemosignaling mechanisms in primates. American Journal of Primatology, 68, 525-544. https://doi.org/10. 1002/ajp.20250

Ezenwa, V. O., \& Williams, A. E. (2014). Microbes and animal olfactory communication: Where do we go from here? BioEssays, 36(9), 847-854. https://doi.org/10.1002/bies.201400016
Fontani, S., Tanteri, G., Vaglio, S., Delfino, G., \& Moggi-Cecchi, J. (2014). Histology of the suprapubic and anogenital cutaneous glands in male cotton-top tamarins (Saguinus oedipus). Folia Primatologica, 85, 109-118. https://doi.org/10.1159/000360221

Gałuszka, A., Migaszewski, Z. M., \& Namieśnik, J. (2015). Moving your laboratories to the field-Advantages and limitations of the use of field portable instruments in environmental sample analysis. Environmental Research, 140, 593-603. https://doi.org/10.1016/j. envres.2015.05.017

Greene, L. K., \& Drea, C. M. (2014). Love is in the air: Sociality and pair bondedness influence sifaka reproductive signalling. Animal Behaviour, 88, 147-156. https://doi.org/10.1016/j.anbehav.2013. 11.019

Greene, L. K., Grogan, K. E., Smyth, K. N., Adams, C. A., Klager, S. A., \& Drea, C. M. (2016). Mix it and fix it: Functions of composite olfactory signals in ring-tailed lemurs. Royal Society Open Science, 3, 1-10. https://doi.org/10.1098/rsos.160076

Guidelines for the Use of Animals. (2018). Guidelines for the treatment of animals in behavioural research and teaching. Animal Behaviour, 135(1), 1-10. https://doi.org/10.1016/j.anbehav.2017.10.001

Havlíček, J., Fialová, J., \& Roberts, S. C. (2019). How diet affects vertebrate semiochemistry? In C. D. Buesching (Ed.), Chemical signals in vertebrates (Vol. 14, pp. 81-93). Springer.

Havlíček, J., \& Roberts, S. C. (2009). MHC-correlated mate choice in humans: A review. Psychoneuroendocrinology, 34(4), 497-512. https://doi.org/10.1016/j.psyneuen.2008.10.007

Henkel, S., \& Setchell, J. M. (2018). Group and kin recognition via olfactory cues in chimpanzees (Pan troglodytes). Proceedings of the Royal Society B: Biological Sciences, 285(1889), 1-10. https://doi.org/ 10.1098/rspb.2018.1527

Henneken, J., Goodger, J. Q. D., Jones, T. M., \& Elgar, M. A. (2017). Dietmediated pheromones and signature mixtures can enforce signal reliability. Frontiers in Ecology and Evolution, 4(Jan), 1-13. https://doi. org/10.3389/fevo.2016.00145

Heymann, E. W. (2003). Scent marking, paternal care, and sexual selection in callitrichines. In C. B. Jones (Ed.), Sexual selection and reproductive competition in primates: New perspectives and directions (pp. 305-325). American Society of Primatologists.

Heymann, E. W. (2006a). The neglected sense-Olfaction in primate behavior, ecology, and evolution. American Journal of Primatology, 68, 519-524. https://doi.org/10.1002/ajp.20249

Heymann, E. W. (2006b). Scent marking strategies of New World primates. American Journal of Primatology, 68, 650-661. https://doi. org/10.1002/ajp.20258

Jjunju, F. P. M., Giannoukos, S., Marshall, A., \& Taylor, S. (2019). In-situ analysis of essential fragrant oils using a portable mass spectrometer. International Journal of Analytical Chemistry, 2019, 1-11. https://doi.org/10.1155/2019/1780190

Kean, E. F., Bruford, M. W., Russo, I. M., Müller, C. T., \& Chadwick, E. A. (2017). Odour dialects among wild mammals. Scientific Reports, 7(September), 1-6. https://doi.org/10.1038/s41598-017-12706-8

Knapp, L. A., Robson, J., \& Waterhouse, J. (2006). Olfactory signals and the MHC: A review and a case study in Lemur catta. American Journal of Primatology, 68, 568-584. https://doi.org/10.1002/ajp.20253

Kücklich, M., Möller, M., Marcillo, A., Einspanier, A., Weiß, B. M., Birkemeyer, C., \& Widdig, A. (2017). Different methods for volatile sampling in mammals. PLOS One, 12(8), 1-18. https://doi.org/10. 1371/journal.pone. 0183440

Kücklich, M., Weiß, B. M., Birkemeyer, C., Einspanier, A., \& Widdig, A. (2019). Chemical cues of female fertility states in a non-human primate. Scientific Reports, 9(1), 13716. https://doi.org/10.1038/ s41598-019-50063-w

Lazaro-Perea, C., Snowdon, C. T., \& de Fatima Arruda, M. (1999). Scentmarking behavior in wild groups of common marmosets (Callithrix 
jacchus). Behavioral Ecology and Sociobiology, 46(5), 313-324. https:// doi.org/10.1007/s002650050625

Leclaire, S., Jacob, S., Greene, L. K., Dubay, G. R., \& Drea, C. M. (2017). Social odours covary with bacterial community in the anal secretions of wild meerkats. Scientific Reports, 7(1), 1-13. https:// doi.org/10.1038/s41598-017-03356-X

Lledo-Ferrer, Y., Peláez, F., \& Heymann, E. W. (2010). Can overmarking be considered as a means of chemical mate guarding in a wild callitrichid? Folia Primatologica, 81(4), 200-206. https://doi.org/10.1159/000320059

MacDonald, E. A., Fernandez-Duque, E., Evans, S., \& Hagey, L. R. (2008). Sex, age, and family differences in the chemical composition of owl monkey (Aotus nancymaae) subcaudal scent secretions. American Journal of Primatology, 70, 12-18. https://doi.org/10.1002/ajp.20450

Miller, K. E., Laszlo, K., \& Dietz, J. M. (2003). The role of scent marking in the social communication of wild golden lion tamarins, Leontopithecus rosalia. Animal Behaviour, 65, 795-803. https://doi. org/10.1006/anbe.2003.2105

Moraes, I. A., De-Carvalho, M. C. A., de Azevedo Valle, H., Pessoa, V. P., Ferreira, A. M. R., \& Pissinatti, A. (2006). Histology of the sternal and suprapubic skin areas in lion tamarins (Leontopithecus sp. Callitrichidae-Primates). American Journal of Primatology, 1222(68), 1120-1126. https://doi.org/10.1002/ajp.20287

Müller-Schwarze, D. (2006). Chemical Ecology of Vertebrates. Cambridge University Press.

Nagle, H. T., Schiffman, S., \& Gutierrez-Osuna, R. (1998). The how and why of electronic noses. IEEE Spectrum, 35(September), 22-31. https://doi.org/10.1109/6.715180

Oksanen, J., Blanchet, F. G., Friendly, M., Kindt, R., Legendre, P., McGlinn, D., \& Wagner, H. (2019). Vegan: Community Ecology Package. R package version 2.5-6. https://cran.r-project.org/ package=vegan

Penn, D., \& Potts, W. K. (1998). Chemical signals and parasite-mediated sexual selection. Trends in Ecology \& Evolution, 13(10), 391-396. https://doi.org/10.1016/S0169-5347(98)01473-6

PerkinElmer. (2016). Torion T-9 GC/MS product note. http://marketing. govsci.com/TorionT-9_GCMS_OnGSAWEB.pdf

Persaud, K., \& Dodd, G. (1982). Olfactory system using a model nose. Nature, 299(September), 352-355.

Poirier, A. C. (2019). Scent-marking behaviour and semiochemistry in the Callitrichidae. Anglia Ruskin University. https://arro.anglia.ac.uk/id/ eprint/705285

R Core Team. (2020). R: A language and environment for statistical computing. https://www.r-project.org/

Rigaill, L., Suda-Hashimoto, N., Ducroix, L., Mouri, K., Furuichi, T., \& Garcia, C. (2017). Testing for links between female urine odor and male sexual behaviors in Japanese Macaques (Macaca fuscata). International Journal of Primatology, 38(5), 823-837. https://doi.org/ 10.1007/s10764-017-9980-y

Röck, F., Barsan, N., \& Weimar, U. (2008). Electronic nose: Current status and future trends. Chemical Reviews, 108(2), 705-725. https://doi. org/10.1021/cr068121q

Safi, K., \& Kerth, G. (2003). Secretions of the interaural gland contain information about individuality and colony membership in the Bechstein's bat. Animal Behaviour, 65(October), 363-369. https:// doi.org/10.1006/anbe.2003.2067

Scordato, E. S., \& Drea, C. M. (2007). Scents and sensibility: Information content of olfactory signals in the ringtailed lemur, Lemur catta. Animal Behaviour, 73(2), 301-314. https://doi.org/10.1016/j. anbehav.2006.08.006

Scordato, E. S., Dubay, G., \& Drea, C. M. (2007). Chemical composition of scent marks in the ringtailed lemur (Lemur catta): Glandular differences, seasonal variation, and individual signatures. Chemical Senses, 32(5), 493-504. https://doi.org/10.1093/chemse/bjm018

Setchell, J. M., Vaglio, S., Abbott, K. M., Moggi-Cecchi, J., Boscaro, F., Pieraccini, G., \& Knapp, L. A. (2011). Odour signals major histocompatibility complex genotype in an Old World monkey. Proceedings of the Royal Society B, 278(1703), 274-280. https://doi. org/10.1098/rspb.2010.0571

Setchell, J. M., Vaglio, S., Moggi-Cecchi, J., Boscaro, F., Calamai, L., \& Knapp, L. A. (2010). Chemical composition of scent-gland secretions in an Old World monkey (Mandrillus sphinx): Influence of sex, male status, and individual identity. Chemical Senses, 35(3), 205-220. https://doi.org/10.1093/chemse/bjp105

Shen, V. K., Siderius, D. W., Krekelberg, W. P., \& Hatch, H. W. (2014). NIST Standard Reference Simulation Website, NIST Standard Reference Database Number 173.

Sikes, R. S., \& the Animal Care and Use Committee of the American Society of Mammalogists. (2016). 2016 Guidelines of the American Society of Mammalogists for the use of wild mammals in research and education. Journal of Mammalogy, 97(3), 663-688. https://doi. org/10.1093/jmammal/gyw078

Smith, A. C., Knogge, C., Huck, M., Lottker, P., Buchanan-Smith, H. M., \& Heymann, E. W. (2007). Long-term patterns of sleeping site use in wild saddleback (Saguinus fuscicollis) and moustached tamarins (S. mystax): Effects of foraging, thermoregulation, predation, and resource defense constraints. American Journal of Physical Anthropology, 134, 340-353. https://doi.org/10.1002/ajpa

Smith, T. D., Garrett, E. C., Bhatnagar, K. P., Bonar, C. J., Bruening, A. E., Dennis, J. C., Kinznger, J. H., Johnson, E. W., \& Morrison, E. E. (2011). The vomeronasal organ of New World monkeys (platyrrhini). Anatomical Record, 294(12), 2158-2178. https://doi.org/10.1002/ar.21509

Smith, T. E., \& Abbott, D. H. (1998). Behavioral discrimination between circumgenital odor from peri-ovulatory dominant and anovulatory female common marmosets (Callithrix jacchus). American Journal of Primatology, 46(4), 265-284. https://doi.org/10.1002/(SICI)10982345(1998)46:4<265::AID-AJP1>3.0.CO;2-D

Smith, T. E., Schaffner, C. M., \& French, J. A. (1997). Social and developmental influences on reproductive function in female Wied's black tufted-ear marmosets (Callithrix kuhli). Hormones and Behavior, 31(2), 159-168. https://doi.org/10.1006/hbeh.1997.1380

Smith, T. E., Tomlinson, A. J., Mlotkiewicz, J. A., \& Abbott, D. H. (2001). Female marmoset monkeys (Callithrix jacchus) can be identified from the chemical composition of their scent marks. Chemical Senses, 26(5), 449-458. https://doi.org/10.1093/chemse/26.5.449

Snowdon, C. T., Ziegler, T. E., Schultz-Darken, N. J., \& Ferris, C. F. (2006). Social odours, sexual arousal and pairbonding in primates. Philosophical Transactions of the Royal Society of London, Series B: Biological Sciences, 361(1476), 2079-2089. https://doi.org/10.1098/ rstb.2006.1932

Spence-Aizenberg, A., Kimball, B. A., Williams, L. E., \& Fernandez-Duque, E. (2018). Chemical composition of glandular secretions from a pairliving monogamous primate: Sex, age, and gland differences in captive and wild owl monkeys (Aotus spp.). American Journal of Primatology, 80(2), e22730. https://doi.org/10.1002/ajp.22730

Sussman, R. W. (2003). Primate ecology and social structure (Vol. 2: New World Monkeys). Pearson Custom Publishing, Needham Heights, MA.

Thompson, C. L., Bottenberg, K. N., Lantz, A. W., de Oliveira, M. A. B., Melo, L. C. O., \& Vinyard, C. J. (2020). What smells? Developing infield methods to characterize the chemical composition of wild mammalian scent cues. Ecology and Evolution, 10(11), 4691-4701. https://doi.org/10.1002/ece3.6224

Vaglio, S. (2009). Chemical communication and mother-infant recognition. Communicative and Integrative Biology, 2(3), 279-281. https://doi. org/10.4161/cib.2.3.8227

Vaglio, S., Minicozzi, P., Romoli, R., Boscaro, F., Pieraccini, G., Moneti, G., \& Moggi-Cecchi, J. (2016). Sternal gland scent-marking signals sex, age, rank, and group identity in captive mandrills. Chemical Senses, 41(2), 177-186. https://doi.org/10.1093/chemse/bjv077

Washabaugh, K., \& Snowdon, C. T. (1998). Chemical communication of reproductive status in female cotton-top tamarins (Saguinus oedipus 
oedipus). American Journal of Primatology, 45(4), 337-349. https://doi. org/10.1002/(SICI)1098-2345(1998)45:4<337::AID-AJP2>3.0.CO;2-X

Watsa, M. (2013). Growing up Tamarin: Morphology, reproduction, and population demography of sympatric free-ranging Saguinus fuscicollis and S. imperator. Washington University in St Louis. https://doi.org/ 10.7936/K7DB7ZTD

Watsa, M., Erkenswick, G., Halloran, D., Kane, E. K., Poirier, A., Klonoski, K., \& Zuñiga, A. (2015). A field protocol for the capture and release of callitrichids. Neotropical Primates, 22(2), 59-68.

Watsa, M., Erkenswick, G., \& Robakis, E. (2017). Modeling developmental class provides insights into individual contributions to infant survival in callitrichids. International Journal of Primatology, 38(6), 1032-1057. https://doi.org/10.1007/s10764-017-9995-4

Weiß, B. M., Kücklich, M., Thomsen, R., Henkel, S., Jänig, S., Kulik, L., Birkemeyer, C., \& Widdig, A. (2018). Chemical composition of axillary odorants reflects social and individual attributes in rhesus macaques. Behavioral Ecology and Sociobiology, 72(4), 65. https://doi. org/10.1007/s00265-018-2479-5

Weiß, B. M., Marcillo, A., Manser, M., Holland, R., Birkemeyer, C., \& Widdig, A. (2018). A non-invasive method for sampling the body odour of mammals. Methods in Ecology and Evolution, 9(2), 420-429. https://doi.org/10.1111/2041-210X.12888

Winternitz, J. C., \& Abbate, J. L. (2015). Examining the evidence for major histocompatibility complex-dependent mate selection in humans and nonhuman primates. Research and Reports in Biology, 6, 73-88. https://doi.org/10.2147/RRB.S58514

Wyatt, T. D. (2014). Pheromones and animal behaviour (2nd ed.). Cambridge University Press. https://doi.org/10.1017/CBO9781139030748

Wyatt, T. D. (2015). The search for human pheromones: The lost decades and the necessity of returning to first principles. Proceedings of the Royal Society B: Biological Sciences, 282, 1-9. https://doi.org/10.1098/ rspb.2014.2994

\section{SUPPORTING INFORMATION}

Additional Supporting Information may be found online in the supporting information tab for this article.

How to cite this article: Poirier AC, Waterhouse JS, Watsa M, et al. On the trail of primate scent signals: A field analysis of callitrichid scent-gland secretions by portable gas chromatography-mass spectrometry. Am J Primatol. 2021;e23236. https://doi.org/10.1002/ajp.23236 